\title{
Characteristics of Celiac Disease in Childhood of Eastern Algeria
}

\author{
Nousseiba Abed ${ }^{1 *}$,Nour el houda Cherouat ${ }^{2}$, Hanane Mohammedi $^{2}$, Abdelkader Rouabah $^{1}$, \\ Leila Rouabah ${ }^{1}$ \\ ${ }^{1}$ Laboratory of Cellular and Molecular Biology, Faculty of Nature and Life Sciences, University of Brothers \\ Mentouri, 25000, Constantine - Algeria \\ ${ }^{2}$ Department of Biochemistry and Biological Cellular and Molecular, Faculty of Nature and Life Sciences, \\ University of Brothers Mentouri, 25000, Constantine - Algeria
}

\begin{abstract}
Objective: To determine the symptoms, associated pathologies and features of Childhood Celiac Disease in the population of eastern Algeria.

Study: The retrospective study was conducted in Pediatric Specialized Hospital of Sidi Mabrouk - Constantine. This study was based on data of 100 patients.

Results: In this sample, we noted a diversity of signs and associated pathology, a variety of digestive symptoms represented mainly by abdominal pain (59\%), chronic diarrhea (51\%) and vomiting (48\%). Extra-digestive symptoms represented mainly by palorous skin and mucosa, delayed status and weight and stagnation of the weight with the percentages: $69 \%, 53 \%$ and $51 \%$ respectively. In addition, we noted biological disorders and a series associated pathologies namely: anemia (29\%) and mouth ulcers (6\%).

Conclusion: Childhood Celiac Disease (CD) of eastern Algeria can be expressed by a variety of digestive symptoms and gastrointestinal disorders (GI) in addition to a series of associated conditions.
\end{abstract}

Key words: Infant Celiac Disease, Symptoms, Associated Conditions, Eastern Algeria

\section{Introduction}

Celiac disease is a chronic inflammatory autoimmune enteropathy (AIE), characterized by intestinal villous atrophy and inflammatory infiltration of the duodenum and jejunum, induced by prolamins (gluten) [1, 2].

The prevalence of the disease in the world is estimated at $1 \%$ in the general population and increases to $33 \%$ in populations "at risk" [3].

Celiac disease promotes the occurrence of many complications mainly malignant lesions [4].The overall risk of death for a person with celiac disease is increased by $39 \%$ compared to the general population [5]. The only effective

\footnotetext{
* Corresponding author: Nousseiba Abed,
} nousseibaabed@gmail.com

(cc) BY-NC-ND $\odot 2019$ Nousseiba Abed et al., published by De Gruyter Open. This work was licensed under the Creative Commons Attribution-NonCommercialNoDerivs 3.0 License treatment for this disease is a strict gluten-free diet for a life [6].

The last two decades, the face of celiac disease has changed significantly. Thus, it has gone from being a rare disease affecting mainly children in a classic clinical presentation to one of the most common chronic disease in the world that can be diagnosed at any age and includes mainly atypical expressions [7].

Completing the clinical picture, associated pathology and the peculiarities of this disease in infant and children in our population, we report the results of a retrospective study in the region of eastern Algeria.

\section{Material and Methods}

We performed a retrospective study in Pediatric Specialized Hospital of Sidi Mabrouk Constantine, which is located in eastern of Algeria. This study was based on data of 100 patients with celiac disease who were identified 
using exploitation card. We applied, as criteria for inclusion of individuals with celiac disease, both sexes having less than 18 years. Exclusion criteria are respectively absence of celiac disease and subjects aged of 18 years and more. SPSS version 20 for Windows was used for descriptive statistics. Results were calculated as frequencies (\%), means and standard deviations (SD).

We used Chi-square test to study correlations between histology grade and serological tests, respectively different manifestations of disease, in addition to the correlation between age and histology grade, respectively comorbidities. The criterion for statistical significance was $p<0.05$.

\section{Results and Discussions}

The sample consists of $60 \%$ females and revealed a female / male ratio of $1.5 \%$. The median age of children was $5.2 \pm 3.67$ years (range, 1-16 years), with a body mass index of $15.52 \pm 2.28 \mathrm{~kg} / \mathrm{m} 2,93 \%$ of the patients are underweight. The median age of the children at diagnosis was $3.50 \pm 2.578$ years with a minimum of 1 and a maximum of 13 years. $95 \%$ of the patients were breastfed for duration between 6 and 24 months. The average duration of breastfeeding was $9.37 \pm 5.94$ months. The median age of introduction of gluten in the diet was $4.10 \pm$ 1.367 months. It was between 2 and 7 months. We observed consanguinity in $32 \%$ of cases and a presence of similar cases in the family in $42 \%$. We sought in this study all visceral, clinical or morphological manifestations and any pathology associated with celiac disease. Clinical pictures found namely digestive manifestations, extradigestive alone or in association with other diseases. In a series of patients, we have not found asymptomatic forms. The most frequent gastrointestinal symptoms were abdominal pain (59\%) and chronic diarrhea (51\%). Patients suffering from vomiting represent $48 \%$ (Figure 1).

Extra-intestinal symptoms are mainly represented by palorous skin and mucosa, delayed status and weight and stagnation of the weight with the percentages: $69 \%, 53 \%$ and $51 \%$ respectively. Another series of extra-intestinal symptoms found namely weight loss (42\%) and asthenia (32\%) (Figure 2).

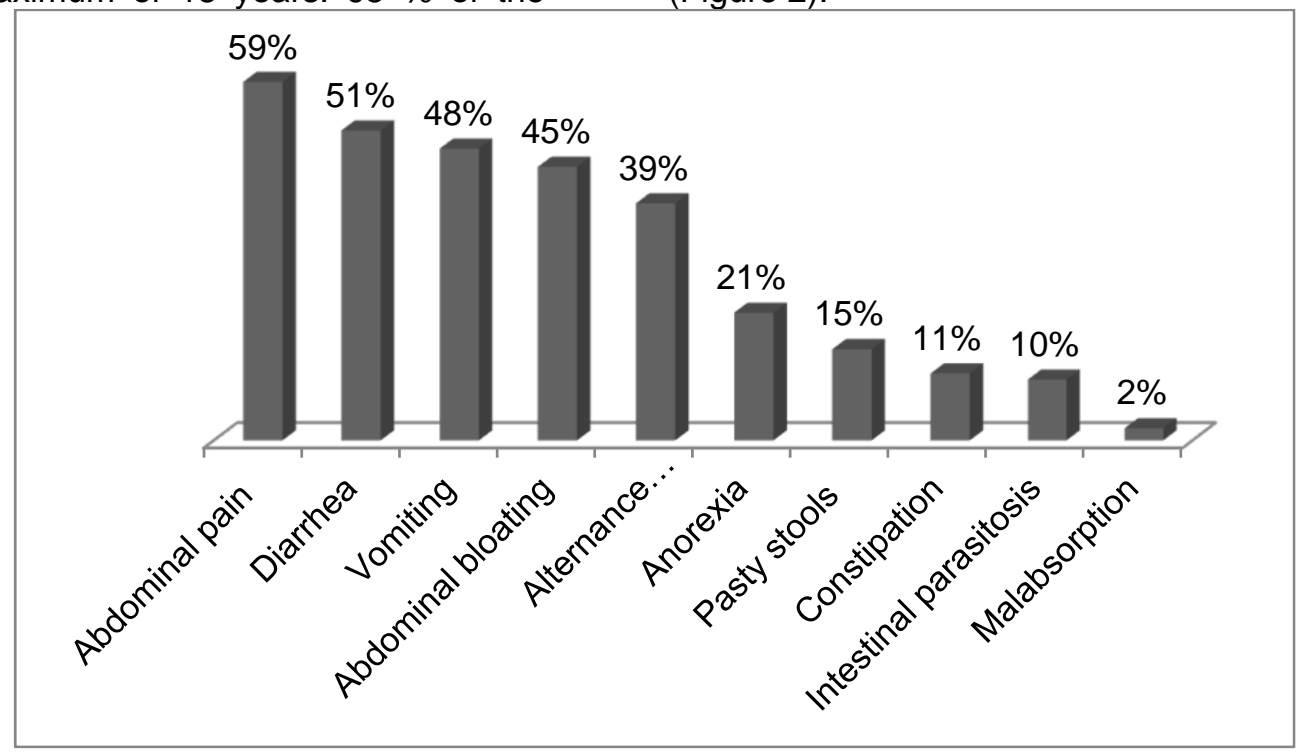

Fig. 1. Distribution of Gastrointestinal Symptoms 


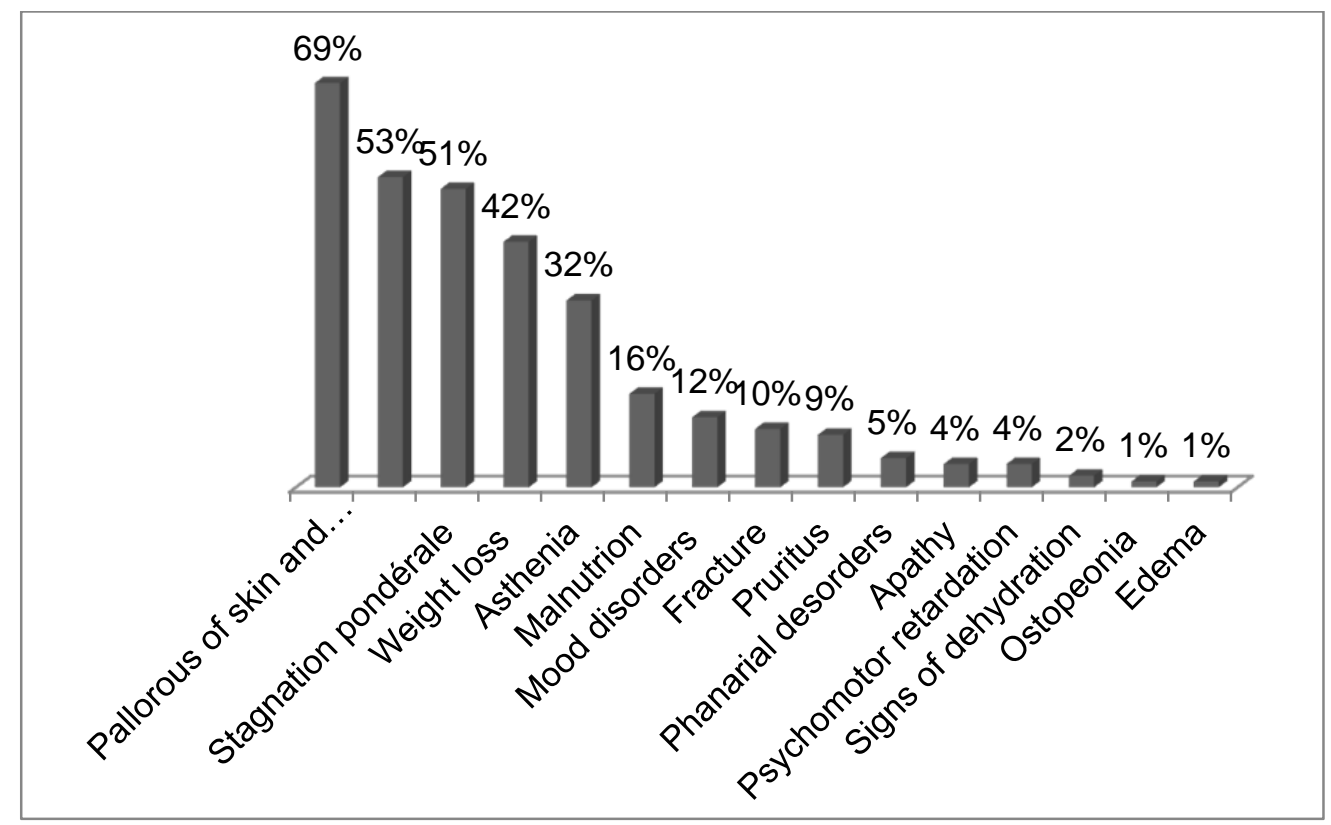

Fig. 2. Distribution of Extra-digestive Symptoms

Our series is marked by the presence of certain pathologies associated with digestive or extradigestive symptoms. There are represented mainly by anemia with a percentage of $29 \%, 6 \%$ of patients have mouth ulcers, dermatitis herpetiformis is presented by $3 \%$ of patients and other pathologies associated with celiac disease are found in (Figure 3).
The results show that celiac disease may be accompanied by disruption of biological tests found essentially in hypoferritinemia (Figure 4). In this sample of patients we find that anti-tissue transglutaminase and anti-endomysial antibody tests presented the highest sensitivity for untreated celiac disease (Figure 5).

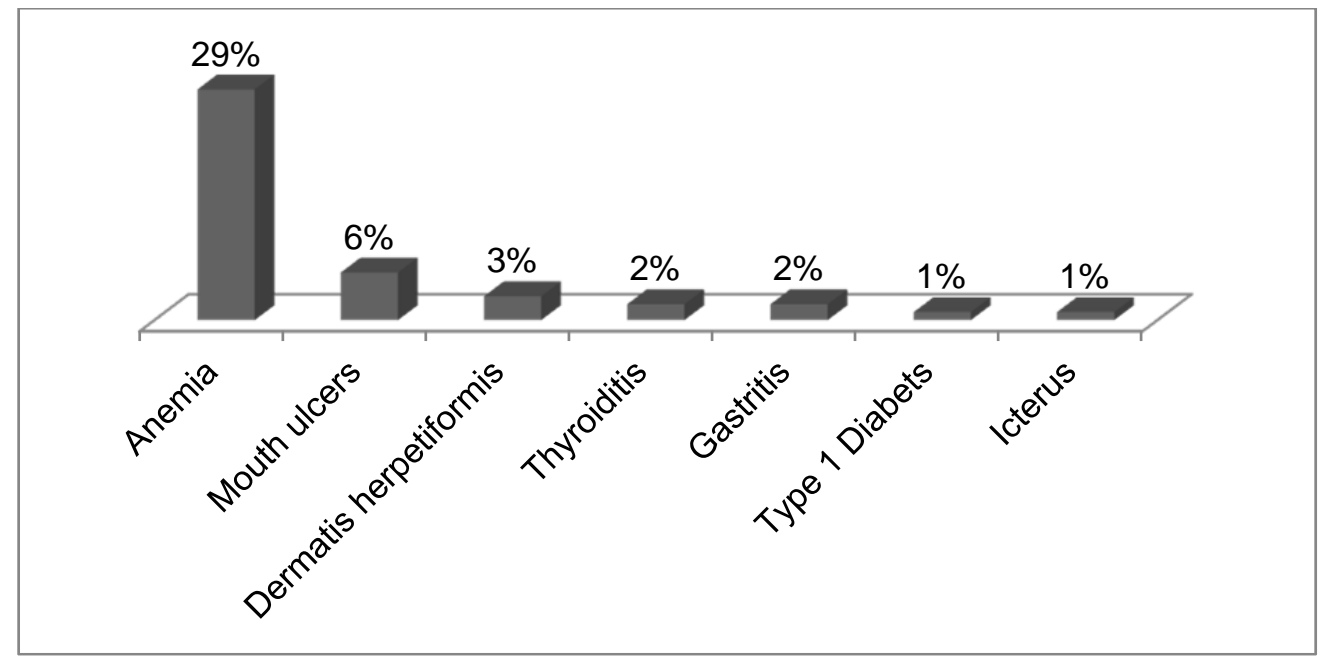

Fig. 3. Distribution of Associated Conditions 


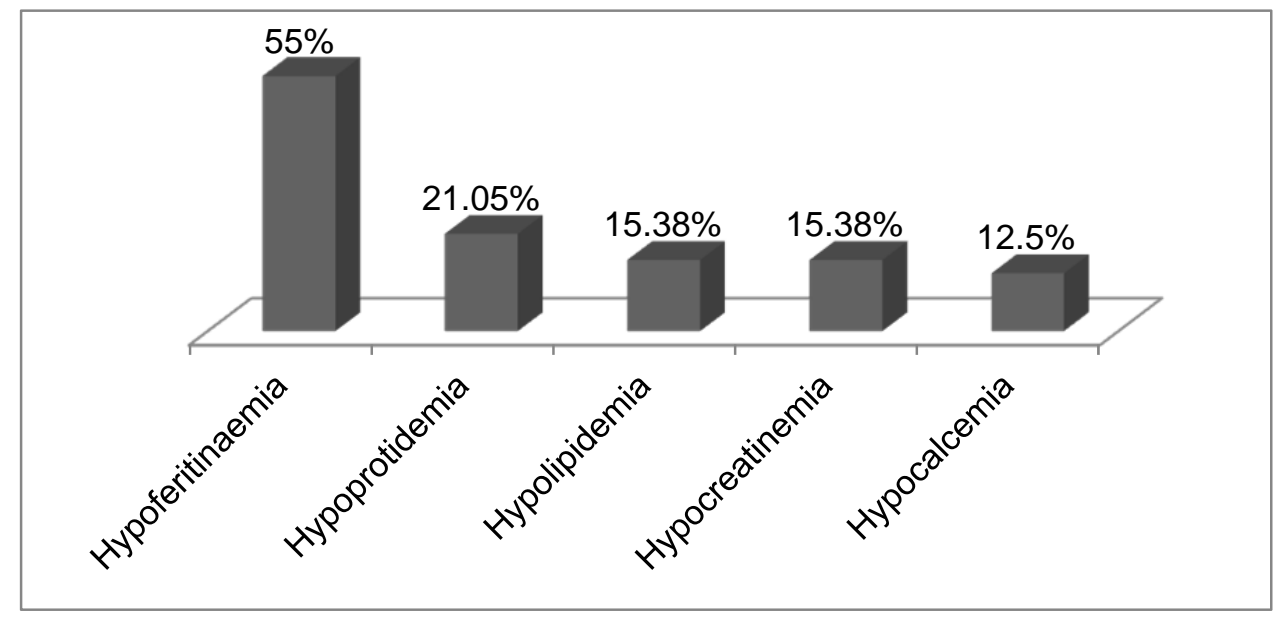

Fig. 4. Disruption of Biological Tests

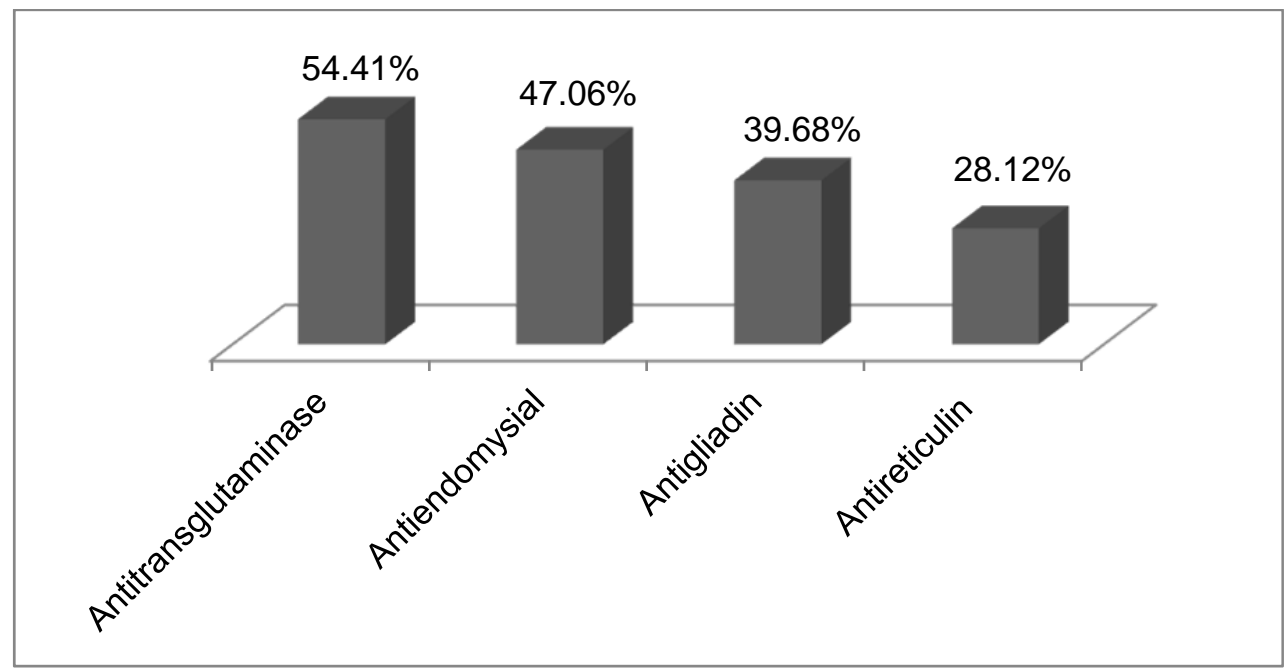

Fig. 5. Disruption of Positivity of Serological Tests

The results of biopsies demonstrated the diagnosis of CD was correct in $100 \%$ of children. We noted three histological grades; total villous atrophy (32\%), subtotal villous atrophy (34\%) and partial villous atrophy (34\%). The gluten-free diet was prescribed in all patients, once the diagnosis is retained. Iron supplementation was prescribed for all patients with anemia, folic acid supplementation was prescribed for 6 patients, metronidazole has been prescribed as antiparasitic treatment in all patients presenting digestive disorders and paraffin oil was prescribed as a treatment for constipation. All patients had the gluten-free diet; the effect of the GFD was spectacular among 100 children with transit disorders, $96 \%$ of them found a normal transit to the first clinical control at the consultation from 3 month of the GFD. $53 \%$ of the children on all of those who had a delayed status and weight had an improvement in the first few months of the diet and 86 in 100 who had a behavioral disorder are improved. $22 \%$ of patients showed histological repair and among 100 serological tests carried out: 22 revealed negative after 2 years under GFD. The other serological tests remained positive but at rates lower than 3 times.

Statistical analysis shows that there was no significant correlation between histology grade and serological tests. It was significantly correlated only with antiendomysial test $(p<0.05)$. The age was not significantly correlated with histology grade, but there is a significant correlation with Intestinal parasitosis, weight loss, malnutrition, mood disoders, pruritus, apathy, signs of dehydration and edema ( $p<0.05)$. On the other hand, there was no significant correlation between histology grade and all symptoms.

Celiac disease was described traditionally as a state of malabsorption, with diarrhea and steatorrhea, appeared in childhood after the introduction of gluten into the diet. It is now 
recognized that his presentation is much more diverse and the diagnosis of $C D$ is based on clinical, serological and histological findings. Clinically, the picture is very variable [8]. It is not only an intestinal disease, but a multi- system disease as well $[9,10]$.

Clinical manifestations revealing this disease have changed in profile over the last 20 years. Today, the classic triad associating steatorrhea, abdominal meteorism and weight loss are not the main reason for consultation. The digestive signs are most often discreet or even absent. Isolated signs of malabsorption may be in the foreground and extra-digestive manifestations of the disease, atypical, may misdiagnosis for several years. Often, digestive and extra-digestive signs are associated making diagnosis even more difficult [11,12].

Celiac disease can occur at any age, there are two frequency peaks: one during childhood, it is early before 5 months especially if the introduction of gluten is premature. Most often, it appears in infant between 6 months and 2 years, the period corresponding to the weaning of breast milk. In these cases, we speak about "infant forms". However, it can occur in children around 9 years old and we speak in this case of "child forms". It can even sometimes reach later, often in adulthood between 20 and 40 years-old and earlier in women than in men, and in this case we speak of "adult forms". The forms with late revelation, after 65 years, are however not exceptional $[4,13,14,15]$. In our study, the median age of children at diagnosis was $3.50 \pm$ 2.578 years.

Most studies on celiac disease report a predominance of female subjects compared to male [16]. This is comparable to our results of female to male ratio of 1.5 .

The literature suggests the protective effect of breastfeeding on the development of celiac disease [17]. However, in this study $95 \%$ of patients were breastfed for duration between 6 and 24 months. The average duration of breastfeeding was $9.37 \pm 5.94$ months.

The classic form of the disease is characterized by the most common typical symptoms like diarrhea with steatorrhea, weight loss, malnutrition, asthenia and abdominal pain in addition to symptoms of malabsorption [4].This data is similar to our results. The most frequent gastrointestinal symptoms noted were abdominal pain (59\%), chronic diarrhea (51\%) and vomiting (48\%). In addition, to a very variable extraintestinal manifestations particularly palorous skin and mucosa (69\%), delayed status (53\%), weight and stagnation of the weight (51\%) weight loss (42\%) and asthenia (32\%). These symptoms may be secondary to mal absorption syndrome or independent of it.

The atypical form of celiac disease is the most common form, made of extra-digestive or digestive but non-specific symptoms [18].

Extra digestive symptoms related to malabsorption may be in the foreground, mainly bone pain, fractures, tetany and muscle cramps, hypocalcemia and / or hypomagnesemia,[19]. The disruptions of biological tests are iron deficiency "anemia", folate deficiency, vitamin B12 deficiency, vitamin K-dependent factors (II, VII, IX and $X)$ deficiency, hypoalbuminemia, hypocalcemia, hypomagnesemia and zinc deficiency [20]. In this study we recorded that $29 \%$ of patients suffer from anemia, $6 \%$ have mouth ulcers and dermatitis herpetiformis is presented by $3 \%$ of patients, in addition to thyroiditis, type 1 diabetes and icterus.

Five conditions have been shown to be significantly associated with celiac disease: Insulin dependent diabetes mellitus (IDDM), thyroiditis with dysthyroidism, Selective immunoglobulin A (IgA) deficiency (SIgAD), dermatitis herpetiformis $(\mathrm{DH})$, and Primary biliary cirrhosis (PBC) $[19,21]$

\section{Conclusions}

Our results shows that celiac disease in children of eastern Algeria can be noticed through a variety of digestive symptoms mainly by chronic diarrhea and abdominal pain, and non gastrointestinal disorders which are mainly represented by palorous skin and mucosa, delayed status and weight, stagnation of weight in addition to a series of associated conditions.

A prospective large-scale and multicenter study should be carried out in the future to further validate our findings.

\section{Acknowledgements}

This section should present sources of supporting funding of the work and list those who contributed or helped during the research (analysis, data interpretation, language, writing, etc.).

\section{References}

1. Sollid, L.M. (2000). Molecular basis of celiac disease. Annu Rev Immunol., 18:53-81, https://doi.org/10.1146/annurev.immunol.18.1.53

2. Farrell, R.J, \& Kelly, C.P. (2002). Celiac sprue.NEngl JMed., 346:180-188, https://doi.org/10.1056/NEJMra010852

3. Pinier, M. (2010). A new treatment strategy for celiac disease based on sequestering polymers. Thesis of Philosophae Doctorate in Pharmaceutical Sciences, University of Montreal.

4. Weber, A. (2012). Celiac disease: physiopathology and treatment "guide" advice for the pharmacist. Thesis of Doctorate in Pharmacy. University of Lorraine. 
5. Ludvigsson, J.F., Montgomery, S.M., Ekbom, A., Brandt, L., \& Granath, F. (2009). Small-intestinal histopathology and mortality risk in celiac disease. JAMA, https://doi.org/10.1001/jama.2009.1320

6. Hill, I.D., Dirks, M.H., Liptak, G.S., Colletti, R.B., Fasano, A., Guandalini, S., Hoffenberg, E.J., Horvath, K., Murray, J.A., Pivor, M., \& Seidman, E.G. (2005). Guideline for the Diagnosis and Treatment of Celiac Disease in Children: Recommendations of the North American Society for Pediatric Gastroenterology, Hepatology and Nutrition J Pediatr Gastroenterol Nutr, 40(1):1-19.

7. El Yaouti Siham. (2010). Celiac disease in children (about 266 cases). Thesis of Doctorate in medicine. Sidi Mohamed Ben Abdallah University.

8. Tkoub, E.M. (2008). Celiac disease of the adult. Revue française d'allergologie etd'immunologie clinique, $48: 27-31$.

9. Sollid Ludvig, M. (2002). Coeliac Disease: Dissecting a Complex Inflammatory Disorder. Nat Rev Immunol. 2(9):647-55, https://doi.org/10.1038/nri885

10. Murray, J.A, Van Dyke, C., Plevak, M.F., Dierkhising, R.A., Zinsmeister, A.R. \& Melton $3^{\text {rd }}$ J. (2003). Trends in the Identification and Clinical Features of Celiac Disease in a North .American Community, Clinical Gastroenterology and Hepatology: the Official Clinical Practice. Journal of the American Gastroenterological Association, 1(1), 19-27, https://doi.org/10.1053/jcgh.2003.50004

11. El abkari, M.I., Benelbarhdadi, \& E.A. Essaid. (2006). Celiac disease of the adult. About 39 cases. Médecine du Maghreb, 133 : 49-61.

12. Malamut, G., \& Cellier, C. (2010). Celiac disease, La Revue de médecine interne, 31, 428-433.
13. Bourrillon, A. (2000). Collection for the practitioner. Pédiatrie. 3ème édition, Masson, Paris, $618 \mathrm{p}$.

14. Boudraa, G.W. Hachela., M.B., Berrahal, N., Hamidi, N., \& Touhami, M. (2003). «Interest of serology in the detection of celiac disease. "Scientific days of the ANDRS $\gg$ Journées scientifiques de l'ANDRS : 17 et 18 décembre 2003, 72-75.

15. Bayrou, O. (2001). Celiac disease In: The vademecum of the diagnosis. MMI éditions, Paris, 1261 p.

16. Bouasla. (2011). Prevalence of celiac disease in Constantine (1996-2008) and dietary associated with patients of EHS Sidi Mabrouk of Constantine (2009). Memory of Magister in Food Science. Option: Human Nutrition. Mentouri University. Constantine

17. Garnier-Lengliné, H.G., Malamut, N.C.-B., \& Ruemmele, F.M. (2013). Therapeutic perspectives. Pathologie Biologie, 61(3), e61-e64.

18. Rampertab, S.D., Pooran, N., Brar, P., Singh, P. \& Green, P.H.R. (2006). Trends in the presentation of celiac disease. Am J Med, 119:355.e9-14, https://doi.org/10.1016/j.amjmed.2005.08.044.

19. Collin, P., Reunala, T., Pukkala, E., Laippala, P., Keyriläinen, O. \& Pasternack, A. (1994). Celiac disease-associated disorders and survival. Gut, ;35:1215-1218, http://dx.doi.org/10.1136/gut.35.9.1215. 20. Farell, R.J. \& Kelly, C.P. (2002). Celiac sprue. N. Engl. J. Med, 346:180-188 et 347:446-448, https://doi.org/10.1056/NEJMra010852 .

21. Kingham. J.G. \& Parker, D.R. (1998). The association between primary biliary cirrhosis and Celiac disease: a study of relative prevalences. Gut, 42:120122, http://dx.doi.org/10.1136/gut.42.1.120. 